\title{
Glutathione-S-transferase gene polymorphisms (GSTM1, GSTT1, GSTP1) and idiopathic male infertility: novel perspectives versus facts
}

\author{
Konstantinos P Economopoulos, Theodoros N Sergentanis and Souzana Choussein \\ Journal of Human Genetics (2010) 55, 557-558; doi:10.1038/jhg.2010.89; published online 5 August 2010
}

$I^{n}$ $\mathrm{n}$ this issue of the Journal of Human Genetics, Safarinejad et al. ${ }^{1}$ present an original case-control study globally assessing glutathione-S-transferase gene polymorphisms (GSTM1 present/null, GSTT1 present/ null, GSTP1 Ile105Val) in the context of idiopathic male infertility. This study is the first to address the potential involvement of the GSTP1 Ile105Val polymorphism in idiopathic male infertility; among the original aspects of the study the synergistic effects that the authors suggested between the aforementioned three polymorphisms are worth acknowledging. Specifically, Safarinejad et al. ${ }^{1}$ suggested a positive association between male infertility and GSTM1 null genotype, GSTT1 null genotype and GSTP1 Ile/Ile status, which seem to mutually potentiate the effects of each other.

Under the light of the findings presented by Safarinejad et al. ${ }^{1}$ we performed a metaanalysis of the existing literature on the association between idiopathic male infertility and GSTM1 and GSTT1 polymorphisms. The meta-analysis was performed so as to explore whether the results reported by Safarinejad et al. ${ }^{1}$ are reproducible at the meta-analytical level. Eligible articles were identified by a search of MEDLINE bibliographical database for the period up to 15 June 2010 using combinations of the following keywords: 'glutathione', 'GSTM1', 'GSTT1', 'polymorphism', 'genotype', 'idiopathic', 'male infertility', 'men'. Language

KP Economopoulos, TN Sergentanis and S Choussein School of Medicine, University of Athens, Athens, Greece and Society of Junior Doctors, Athens, Greece. E-mail: economopoulos@sni.gr restrictions were not used and two investigators (KPE and TNS), working independently, searched the literature and extracted data from each eligible case-control study. On the basis of the genotype frequencies in cases and controls, crude odds ratios (OR) and their s.e. were calculated. The ORs pertained to (i) null genotype carriers vs present (positive) genotype carriers concerning GSTM1, (ii) null genotype carriers vs present (positive) genotype carriers concerning GSTT1. After the assessment of heterogeneity, the random effects (DerSimonian Laird) model was used to calculate the pooled ORs. Between-study heterogeneity and between-study inconsistency were assessed by using Cochran $Q$-statistic and by estimating $I^{2}$, respectively. Evidence of publication bias was determined using Egger's formal statistical test and by visual inspection of the funnel plot. For the interpretation of Egger's test, statistical significance was defined as $P<0.1$. In addition, sensitivity analysis excluding studies on Chinese subjects was performed so as to obtain an estimate pertaining to Caucasian populations. Metaanalysis was performed using the STATA 10.0 'metan' command (STATA, College Station, TX, USA).

Out of the 168 abstracts retrieved through the search criteria, 161 were irrelevant and one was excluded as a case-only study. ${ }^{2}$ Six case-control studies ${ }^{1,3-7}$ were eligible concerning GSTM1 genotype (912 male infertility cases, 859 controls) and five casecontrol studies ${ }^{1,4,6-8}$ were eligible concerning GSTT1 genotype (862 male infertility cases, 755 controls). All studies were conducted on Caucasian subjects except for one $e^{6}$ in the case of GSTM1 polymorphism and two ${ }^{6,8}$ in the case of GSTT1 polymorphism.

GSTM1 null genotype was associated with male infertility only at a borderline level of significance (pooled $\mathrm{OR}=1.28,95 \%$ confidence interval, CI: $0.96-1.70, \quad P=0.09$, Figure 1a). In contrast, GSTT1 null genotype was not associated with male infertility (pooled $\mathrm{OR}=0.98, \quad 95 \% \quad \mathrm{CI}: \quad 0.50-1.89$, $P=0.94$, Figure $1 \mathrm{~b}$ ). At the sensitivity analysis, the borderline association implicating GSTM1 null genotype status vanished (pooled $\mathrm{OR}=1.31, \quad 95 \% \quad \mathrm{CI}: \quad 0.92-1.85$, $P=0.14$ ), whereas the null association concerning GSTT1 was replicated (pooled $\mathrm{OR}=0.58, \quad 95 \% \quad$ CI: $0.16-2.14, \quad P=0.42)$. Despite the low power of the Egger's test, marginal publication bias was observed in the case of GSTT1 polymorphism $(P=0.10)$, but not in the case of GSTM1 polymorphism $(P=0.60)$. Summarizing the above, it can be shed that the results by Safarinejad et al. ${ }^{1}$ do not seem reproducible at the meta-analytical level for the time being. As a result, further studies with substantially larger sample size seem mandatory in order to yield a clear picture of this controversial field.

Although the study by Safarinejad et al. ${ }^{1}$ may open interesting perspectives in the understanding of male infertility, a variety of notions seem worth commenting. Safarinejad et al. ${ }^{1}$ have portrayed the Ile allele as a risk factor for male infertility; this suggestion may seem of questionable importance due to a variety of reasons. First, the Ile allele is by far more prevalent than the Val allele in the human population; it seems worth asking how the infertility-generating Ile allele became more frequent in the male population 

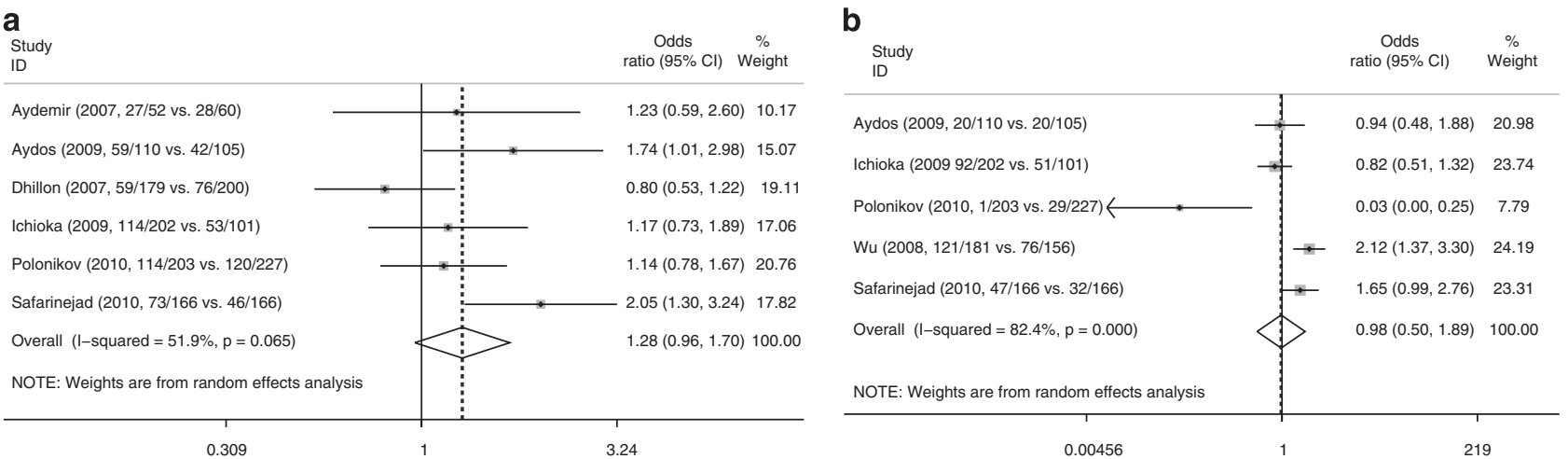

Figure 1 Forest plot for the overall association between (a) null GSTM1 genotype, (b) null GSTT1 genotype and idiopathic male infertility. Each study is shown by the point estimate of the odds ratio (OR) (the size of the square is proportional to the weight of each study) and $95 \%$ confidence interval (CI) for the OR (extending lines); the pooled OR and $95 \% \mathrm{CI}$ have been appropriately derived from random effects model. The study ID consists of the last name of the first author of the study and a parentheses including the year of publication and the proportion of null genotype status of idiopathic male infertility patients versus the proportion of null genotype status of controls.

as the principles of evolutionary genetics dictate that such alleles should become extinct in the successive generations. Indeed, close comparative inspection of the OR and $95 \%$ CI of the double null (GSTM1, GSTT1) status and triple variant (GSTM1, GSTT1, GSTP1) status, that is, 2.92 (1.57-5.54) and 4.45 (1.59-12.24), reveals significant overlapping of confidence limits. In other words, the role of GSTP1 Ile105Val may still remain a controversial issue. At any case, the fact that GSTP1 Ile/Ile did not differ from GSTP1 Val/Val $(P=0.47)$ indicates that the described effect was rather surprisingly due to heterozygous carriers. The functional relevance of this finding remains elusive and dictates that larger studies should be performed, as the study by Safarinejad et al. has only included nine Val/Val subjects. Nevertheless, it should be kept in mind that recent meta-analyses on the field of cancer ${ }^{9,10}$ have pointed to the Val allele as a risk factor for carcinogenesis, and have not supported any aggregating effect of Ile/Ile genotype.
In conclusion, Safarinejad et al. ${ }^{1}$ have opened a debate regarding the relevance of GSTP1 Ile105Val status in male infertility. Given the aforementioned arguments and the results of the present meta-analysis, we point to the need for accumulation of data regarding GSTM1, GSTT1 and GSTP1 polymorphisms; any definitive conclusions for the time being would be premature.

1 Safarinejad, M. R., Shafiei, N. \& Safarinejad, S. The association of glutathione-S-transferase gene polymorphisms (GSTM1, GSTT1, GSTP1) with idiopathic male infertility. J Hum Genet. 55, 565-570 (2010).

2 Finotti, A. C., Costa, E.S.R.C, Bordin, B. M, Silva, C. T \& Moura, K. K. Glutathione $S$-transferase $M 1$ and $T 1$ polymorphism in men with idiopathic infertility. Genet Mol Res. 8, 1093-1098 (2009).

3 Aydemir, B., Onaran, I., Kiziler, A. R., Alici, B. \& Akyolcu, M. C. Increased oxidative damage of sperm and seminal plasma in men with idiopathic infertility is higher in patients with glutathione S-transferase Mu-1 null genotype. Asian J Androl. 9, 108-115 (2007).

4 Aydos, S. E., Taspinar, M., Sunguroglu, A. \& Aydos, K. Association of CYP1A1 and glutathione S-transferase polymorphisms with male factor infertility. Fertil Steril. 92, 541-547 (2009).

5 Dhillon, V. S., Shahid, M. \& Husain, S. A. Associations of MTHFR DNMT3b 4977 bp deletion in mtDNA and GSTM1 deletion, and aberrant CpG island hypermethylation of GSTM1 in non-obstructive infertility in Indian men. Mol Hum Reprod. 13, 213-222 (2007).

6 Ichioka, K. Nagahama, K. Okubo, K. Soda, T. Ogawa, O. \& Nishiyama, H. Genetic polymorphisms in glutathione $S$-transferase $\mathrm{T} 1$ affect the surgical outcome of varicocelectomies in infertile patients. Asian J Androl. 11, 333-341 (2009).

7 Polonikov, A. V., Yarosh, S. L., Kokhtenko, E. V., Starodubova, N. I., Pakhomov, S. P. \& Orlova, V. S. The functional genotype of glutathione $S$-transferase T1 gene is strongly associated with increased risk of idiopathic infertility in Russian men. Fertil Steril.

8 Wu, Q. F., Xing, J. P., Tang, K. F., Xue, W., Liu, M., Sun, J. H. et al. Genetic polymorphism of glutathione $S$-transferase $\mathrm{T} 1$ gene and susceptibility to idiopathic azoospermia or oligospermia in northwestern China. Asian J Androl. 10, 266-270 (2008).

9 Cote, M. L., Chen, W., Smith, D. W., Benhamou, S., Bouchardy, C., Butkiewicz, D. et al. Meta- and pooled analysis of GSTP1 polymorphism and lung cancer: a HuGE-GSEC review. Am J Epidemiol. 169, 802-814 (2009).

10 Sergentanis, T. N. \& Economopoulos, K. P. GSTT1 and GSTP1 polymorphisms and breast cancer risk: a metaanalysis. Breast Cancer Res Treat. 121, 195-202. 